\title{
DIALÉCTICA Y CONTRADICCIÓN: SUS FUNCIONES HERMENÉUTICAS EN UNA INTERPRETACIÓN DE LA FILOSOFÍA DEL DERECHO DE HEGEL CON SENTIDO ACTUAL
}

Luisa H. Meyer*

SÍNTESE - El término "Dialéctica" hace referencia tanto al movimiento de autodesenvolvimiento del concepto - en este caso el de derecho y, en sentido amplio (tò dikaion) - como a la estructura de ese movimiento, al cual Hegel trata de encuadrar en esquemas provenientes de la Lógica del concepto, especialmente en la triada 'tesis antitesis - sintesis', lo cual potencia la complejidad del texto y dificulta su comprensión. Una hermenéútica esclarecedora de la lógica dialéctica en juego, donde a la contradicción le cabe el papel protagónico, exige tener en cuenta estos aspectos como también el hecho de que Hegel, en su cor.cepción de la Dialéctica asume la herencia de la filosofía aristotélica, tanto como del Idealismo Trascendental moderno, ios que se entremezclan permanentemente en intrincada red de múltiples significaciones. La distinción de todos estos aspectos y de su rol en la exposición de la Filosofia del Derecho es imprescindible, tanto para una interpretación adecuada de su letra y espiritu, como de su significación actual. El presente trabajo se concibe como un intento de contribuir al esciarecimiento de esta cuestión. PALABRAS-CLAVES - Dialética, contraditión, Filosofia del Derecho.
ABSTRACT - The term "dialectic" refers both to the movement of self-development of the concept, in this case that of right, and, in the broad sense "tò dikaion", and to the structure of this movement which Hegel tries to explain using models taken from the logic of concept particularly the triad thesis-antithesis-synthesis. These usages by, and intentions of Hegel make for complicated texts that are often difficult to interpret. An interpretation which sheds more light on the dialectical logic in which the contradiction has the principal role, must take into account these aspects and the fact that Hegel, in conceiving the process of dialectic, incorporates both aristotelean philosophy and its modern idealistic transcendental counterpart, both of which combine and intermingle continuously to form an intricate pattern of different meanings. Distinguishing between these different aspects and their role in an exposition of the Philosophy of Right is indispensable both to an adequate interpretation of its letter and spirit and to an understanding of its present-day meaning. It is the intention of this work to contribute to a clearer understanding of this subject.

KEY WORDS - Dialectic, contradiction, Philosophy of Right.

* Universidad Argentina John F. Kennedy, Buenos Aires.

\begin{tabular}{|l|l|l|l|l|l|}
\hline VERITAS & Porto Alegre & v. 43 & $n^{2} 4$ & Dezembro 1998 & p. 873-883 \\
\hline
\end{tabular}


La función de la Dialéctica en la Filosofía del Derecho de Hegel puede inferirse a partir de lo que él mismo expresa en la Introducción de su obra, especialmente en los $\S \S 30$ a 33 .

En términos generales podemos afirmar que es el método del cual se vale Hegel para exponer la realización del concepto de libertad, objeto éste de la Filosofia del Derecho. La contradicción, según veremos, es un momento de dicho método.

En el. § 31 Hegel denomina Dialéctica al principio energético que desata un proceso de particularización del concepto universal. Este proceso tiene la particularidad de

1. crear desde sí mismo sus propias determinaciones. Por lo cual preferimos hablar de autodesenvolvimiento en vez de desarrollo.

2. Este proceso atraviesa diferentes estadios; pero en la transición de un estadio a otro permanece siendo él mismo.

El sujeto de este proceso de autodesenvolvimiento, en su meollo estructural, permanece él mismo en y a través de las difversas formas que alcanza en los diferentes momentos o estadios del proceso de desenvolvimiento. El autodesenvolvimiento, por definición no puede ser externo sino que es interno, es gradual y afecta a toda la estructura del sujeto del desenvolvimiento mismo.

3. Estos estadios no deben ser interpretados sólo como determinaciones conceptuales, que alguien pudiese efectuar, por ej. en un análisis conceptual, sino que son configuraciones reales (§ 32); es decir, el proceso produce, genera, algo. Aquí radica, a mi juicio, la mayor dificultad en la exposición de la Filosofía del Derecho. La cuestión es compleja y requiere, para su esclarecimiento, de una tematización de sus supuestos:

(I). Noción de espíritu del Idealismo Trascendental.

(II). Metafísica teleológica de Aristóteles.

(III). Combinación de ambos en la configuración del esquema que orienta la forma de exposición del objeto de la Filosofia del Derecho.

Hegel asume la concepción del espiritu del idealismo, en tanto potencia estructuradora de realidad y trata de llevarla a su máxima expresión, pues espera de ella una verdadera revolución en el pensar. Se concibe al espíritu no sólo con capacidad de comprensión sino también y, ante todo, con capacidad espontánea de insertarse en la realidad, de estructurarla. Recordemos que tanto en las Lecciones sobre Filosofia del Derecho que dicta en Heidelberg en 1817/18, como en la correspondiente Sección de la Enciclopedia que se ocupa de la teoría del derecho, Hegel usa para este tema, junto al título 'Filosofía del Derecho' la expresión 'Espíritu Objetivo'.' Esta concepción del espíritu es la base fundamental que permite a Hegel desarrollar la teoria del concepto que es capaz de objetivarse en la

1 Cfr. Die Philosophie des Rechts, Mitschrf. v. P. Wann., Heidelberg 1817, § 1, Anm. "Der Name des Naturrechts verdient aufgegeben und durch die Benennung "Philosophische Rechtslehre" oder, wie es sich auch zeigen wird, "Lehre vom Objektiven Geist" ersetzt zu werden. 
realidad llegando a constituir la estructura racional inmanente de la misma. Esto no se da abruptamente de una vez, sino en un proceso gradual en el cual el sujeto del mismo permanece idéntico a pesar de los cambios que produce. El esquema, que toma de la Lógica del concepto, es, a grandes rasgios el siguiente: el concepto, que es subjetivo, se configura primeramente en la objetividad para luego, desde la objetividad, regresar a sí mismo, primeramente como "Idea" y finalmente como "Idea absoluta". Objetividad es aquí el modo de ser del concepto, modo en el cual éste se halla, al mismo tiempo, inmerso en la realidad, como constituyendo su estructura inmanente. ${ }^{2}$

Así en el marco de esta concepción, también el concepto del derecho racional tendría la capacidad de configurar, de modo esencial, las relaciones humanas; y a la Filosofia del Derecho le correspondería la tarea de exponer el modo como este concepto racional estructura dicha realidad. ${ }^{3}$

Para explicar cómo funciona la capacidad estructuradora de realidad del concepto en la historia, en tanto ámbito de efectuación de esa capacidad, Hegel distingue en ella estadios de efectuación de la libertad, y atribuye a estos estadios, a su vez, respectivos estadios de realización del concepto de derecho. Cada estado de derecho objetivo corresponde a un estadio del concepto - subjetivo - de derecho.

\section{II}

Debido a la imbricación permanente de ambas líneas de pensamiento debemos rastrear ya desde aquí el origen aristotélico del pensamiento de Hegel.

La distinción de estadios en el proceso de objetivación del concepto supone no sólo que este proceso es gradual, sino también que se halla orientado a un fin: el de la forma estructural final y acabada. Y por eso Hegel se esfuerza en mostrar que lo que se halla dado primeramente como concepto no desenvuelto o como forma meramente interna es comprendido en su significado acabado recién cuando se puede considerar a su forma finai como realidad efectiva, es decir, en su estadio culminante.

La exposición de la Filosofia del Derecho en la forma estructural de estadios se entiende desde la teoría del movimiento del concepto. La espontaneidad de la voluntad racional y libre en y para sí es el principio energético de la realización de la libertad, también, en estadios; cada uno de éstos son estadios de la realización del concepto de derecho y cada estadio de la realización del derecho constituye cada una de las tres partes de la Filosofía del Derecho: Derecho Abstracto, Moralidad y Eticidad.

Si bien en la base de la teoría del desenvolvimiento del concepto del derecho se halla la concepción del espíritu propia del Idealismo, según la cual éste se impone en la realidad estructurándola a su semejanza, debemos decir también que para llevar a expresión esta concepción Hegel, que porta también siempre la impronta de los clásicos griegos, se vale de la filosofía aristotélica, de marcada ori-

2 Cfr. Enciclopedia $\S 304$

3 Cfr. Enc. 3a. Ed. § 385; cfr. supra. 
entación teleológica y expresa en un modelo orgánico la teoría del autodesenvolvimiento gradual del concepto desde su estructura originaria hacia su forma estructural final.

Para el Hegel que sigue a Aristóteles, el concepto es principio interno, forma que es, primeramente, sólo proyecto, posibilidad o potencia pero que, como tal, es principio de movimiento que inicia un proceso que culmina en una forma articulada acabada (Eidos) precisamente porque ésta estaba ya, desde el comienzo, como fin del proceso de desenvolvimiento y, por lo tanto como hilo orientador del mismo. Por eso el concepto que es forma inmanente en la materia tiene también un carácter dinámico $\mathrm{o}$, mejor dicho, es principio dinámico, generador de movimiento hacia la forma final articulada (Eidos) o Télos. Por eso el concepto es también potencia o forma que se desarrolla hacia la Idea - aquí la del derecho.

Cabe entonces la pregunta de si Hegel simplemente reproduce el modelo de orientación teleológica o si éste asume en Hegel una forma nueva, a pesar de su fidelidad a su origen.

Para el Hegel que lleva, desde hace ya bastante tiempo, la impronta del Idealismo la filosofía sólo puede tener como objeto de consideración el concepto puro, y, por lo tanto, también debe tomar en consideración solamente el desenvolvimiento inmanente del concepto, sin admitir nada exógeno al mismo. De este modo, al aplicar el modelo teleológico al proceso de autodesenvolvimiento del concepto puro que, como tal, debe estar libre de todo contenido empírico, Hegel tiene que liberar a éste de toda connotación teleológica natural y con ello surge un nuevo estado de cosas, del cual, si se derivaran las consecuencias, desde el punto de vista especulativo, se podría esperar la "revolución en el pensar" que Hegel espera ya antes de Jena. En su carta a Schelling del 16 de abril de 1795 Hegel le escribe a éste lo siguiente: "Vom Kant - System und dessen höchster Vollendung erwarte ich eine Revolution im Denken, die von Prinzipien ausgehen wird, die vorhanden sind und nur nötig haben, allgemein bearbeitet auf alles bisherige Wissen angewandt zu werden. Fichtes Wissenschaftslehre nehme ich mir vor, auf den Sommer zu studieren".

Qué le brinda a Hegel la esperanza de una revolución en el pensar? Al aplicar el modelo teleológico con connotaciones orgánicas de Aristóteles - el cual admite, por lo tanto, un cierto determinismo proveniente del mundo natural - al desenvolvimiento del concepto, todos los rasgos de ese modelo orgánico adquieren nueva forma; adquieren un significado nuevo, análogo y metafórico respecto del original.

En el proceso de desarrollo natural-teleológico de Aristóteles y que le sirve a Hegel de base y modelo para la exposición de su teoría, el resultado o forma final de un proceso de desenvolvimiento es previsible "para nosotros" o "en-si". Pero cuando lo que se expone es el desarrollo del concepto y, por tanto, el de un ser con conciencia, surge una gran diferencia: no podemos anticipar el resultado; no podemos predecirlo como si tuviésemos a la vista su "ser-en-sí" fijo, de manera definitiva. A su "en-si" sólo podemos captarlo al final de su desarrollo. Recién al final de su desarrollo un ser dotado de conciencia será lo que siempre ha sido en sí o para nosotros; al final de su proceso de desenvolvimiento su para sí y su en sí se corresponderán, empero la cuestión de las formas que va adoptando su autocon- 
ciencia racional y libre constituyen una cuestión totalmente abierta. Aquí radica la posibilidad de esa "verdadera revolución en el pensar" que espera Hegel. La razón que se realiza en el elemento de la autoconciencia no sólo es inmanente-presente, sino que además se desenvuelve objetivándose para la conciencia en un devenir que, como tal implica tiempo. El tiempo verdadero, de la conciencia denota "necesariamente" en Hegel, apertura, impredecibilidad etc. e.d. libertad. Por eso puede afirmar "El suelo del derecho es lo espiritual,...y su punto de partida es la voluntad, que es libre..."; ${ }^{4} \mathrm{y}$ en las Lecciones de 1818/18: "La voluntad, en tanto se piensa a sí misma, es fuente del derecho". ${ }^{5} \mathrm{Y}$ los órdenes del derecho y de la moralidad, como las instituciones de la familia, de la sociedad civil y del Estado, constituyen una realidad creada por hombres y su origen y fundamento es el "derecho" en tanto voluntad libre que se ha pensado a sí misma y se ha efectuado. El derecho es, pues, espíritu que se ha puesto a sí mismo en la realidad objetiva o, mejor dicho, ha constituido o estructurado esta realidad. El derecho tiene su fundamento no en la naturaleza externa ni en las determinaciones naturales específicas del ser humano, como pueden ser, por ej. necesidades o impulsos, sino en la libertad. Acorde con la metafísica teleológica de la cual se vale Hegel para exponer su teoria del desenvolvimiento del concepto de derecho, la libertad como autodeterminación de la voluntad es, al mismo tiempo, su fin, (télos), su forma final articulada y, por lo tanto también su destinación.

Pero dicha forma hacia la cual y en la cual se desarrolla la voluntad libre verdadera, su destinación, es la voluntad libre en y para sí. Esta es verdaderamente libre, es "verdadera", recién cuando busca en la autodeterminación su autoidentidad y la encuentra. Esta voluntad se caracteriza, sobre todo, por no tener ya por objeto, fines encontrados "naturalmente", sino que en su libre autodeterminación se remite a su propio concepto como su fin (télos).

Hegel pone aquí claramente en evidencia la herencia kantiana del concepto de autodeterminación de la voluntad.

Esta voluntad también se halla, en alguna medida, determinada por impulsos e inclinaciones y también busca la felicidad; pero, en la satisfacción de los mismos busca, ante todo, su propia libertad - en tanto autoidentidad - en la autodeterminación; y hace de este fin el criterio de su decidir.

El § 28 de la Filosofía del Derecho expresa, en concordancia con el § 8 que la idea de sí, primeramente abstracta de la voluntad libre se transforma, gracias a la determinación del concepto, en "la totalidad de un sistema" que, en tanto substancial, está más allá de la contraposición entre un fin subjetivo y su realización. En la objetividad lograda permanece, al mismo tiempo, cabe si misma.

Este desarrollo debe considerarse desde dos aspectos: tanto en el sentido de que la libertad debe devenir objetiva como sistema racional de sí misma, como en el sentido de que ella es esto, es decir, es tal, en tanto realidad-efectiva inmediata.

Que la realización u objetivación de la libertad aparezca bajo este doble aspecto en Hegel es comprensible también a partir de la concepción teleológica aristotélica que le permite interpretar la efectuación del concepto, por un lado,

4 Cfr. Principios de Filosofia del Derecho, § 4.

5 Lecciones sobre Filosofia del Derecho 1818/19, § 1, Obs.; cfr. supra. 
como el emerger en la realidad externa de la posibilidad que existe primero sólo como mera potencia y, por otro lado, como un curso en el cual la potencia o posibilidad, que existe primeramente sólo como simplicidad inmediata, se configura en un todo articulado.

Hegel conceptúa la voluntad racional según este modelo, como un concepto que tiene el "impulso" de salir de su subjetividad y configurar su ser "ser en si" en existencia objetiva. Entiende también este proceso de autoobjetivación como un crear un todo articulado, que es comparable a un organismo viviente. Esta totalidad articulada, que ha logrado diferenciaciones en sí misma, es la que se halla mentada cuando Hegel habla de "sistema" y cuando considera 'racional' a una 'totalidad' tal justamente porque ella es en sí una forma articulada.

El desenvolvimiento de la idea de libertad o de la voluntad ya libre-en-y-parasí consiste, entonces, en llevar aquello que constituye la esencia de la libertad (el ser-cabe-sí) - en tanto 'contenido substancial de la Idea' - a realidad objetiva en la 'totalidad de su sistema' y en esta realidad lde la Idea lo 'substancial' sería independiente de la contradicción de un fin meramente subjetivo y su realización lo habría superado; e.d. la creación de la totalidad de un sistema ya no sería para la voluntad libre un fin aún por realizar; sino que ella misma habría devenido, en tanto totalidad de su sistema, realidad efectiva objetivada. Y en esta unidad de la autoconciencia de una voluntad libre y la realidad efectiva objetivada de su libertad el ser-cabe-sí de la voluntad libre en y para sí se habría realizado como 'sistema racional de sí mismo' y su libertad habria devenido objeto para ella'. Estamos hablando de un sistema de normas, del derecho.

La definición del derecho como existencia de la voluntad libre significa lo siguiente: cuando una existencia es existencia de la voluntad libre en y para sí que se halla en camino de la realización o en la realidad de su libertad, entonces una pretensión jurídica se halla justificada.

Y la correspondiente definición de 'deber' en las Lecciones de 1817 y 1818 significa que cuando una pretensión tal es justificada mediante ello, de tal modo que esta pretensión es una condición de la libertad de la voluntad y de su realización, entonces todos tienen que atenerse a reconocerla, respetarla y, dado el caso, cumplirla.

Así, a la efectuación del concepto de libertad corresponde la realización del concepto del derecho; de modo tal que ambos procesos desembocan en el reino de la libertad realizada; con lo cual se supone, como lo anticipamos anteriormente, una realización del derecho racional en el mundo histórico, mundo que, en este caso coincide con la idea de la libertad como capacidad de autodeterminación.

\section{III}

La concepción del espíritu propia del Idealismo que Hegel hace suya y que concibe al espíritu no sólo con capacidad de comprensión sino también de efectivización en la realidad dándole a ésta su forma, sienta su impronta también en el esquema estructural que Hegel elige para exponer el objeto de su Filosofia del Derecho; y para él adopta, de la Ciencia de la Lógica el ropaje de la dinámica del concepto. 
El concepto, en este caso el concepto de derecho, va constituyendo la estructura racional inmanente de la realidad social. La dinámica de este concepto traza los lineamientos del plan de exposición de la Filosofía del Derecho, es decir, se convierte en su idea programática o guía regulativa de la exposición del objetonúcleo de la obra: el autodesenvolvimiento del concepto de derecho hacia su realización o forma final acabada y articulada. Hegel presenta en las Lecciones de 1818/19, § 1 el objeto de la Filosofía del Derecho con las siguientes palabras: "El derecho natural tiene el concepto racional del derecho y la realización del mismo o sea, la idea del derecho, por objeto". O sea, se supone como punto de partida del desenvolvimiento una forma originaria o principio, un desenvolvimiento o efectuación en la realidad y una forma final articulada del concepto. Dicho de otro modo, el concepto, subjetivo en una primera instancia, se configura primeramente en objetividad que él mismo estatuye y estructura, para luego desde ésta, regresar a sí mismo como idea. De acuerdo con esto Hegel expone entonces primeramente en la Introducción de la Filosofía del derecho el concepto del derecho en tanto forma del concepto (I). Luego, en la verdadera exposición, ofrece la efectuación articulada o configuración de este concepto en la realidad (II). Y, finalmente, expone la realización de este concepto en la perspectiva del télos de la historia universal (III).

Una vez que Hegel ha establecido como objeto de la Filosofia del Derecho el concepto del derecho y su autodesenvolvimiento y ha trazado, a partir de allí el plan de exposición del mismo trata de hacer plausible su teoría también desde la forma que elige para exponerla: la forma de exposición debe ser adecuada al contenido. También ella ha de mostrar el autodesenvolvimiento del concepto de derecho que sigue este derrotero. La concepción dinámica del autodesenvolvimiento del concepto de derecho es la que da la pauta de cómo se ha de exponer metódicamente, de acuerdo al sistema, el objeto de la Filosofia del Derecho.

Se presenta aquí una exigencia tan estricta como ineludible para una filosofía como la de Hegel que pretende situarse, sin renunciamientos, en el marco del Idealismo: en todas las perspectivas posibles de consideración de un objeto, como en cada uno de los estadios de consideración del mismo, la filosofía debe abstenerse de tener en cuenta meras opiniones que, como tales, no hacen al concepto mismo del objeto.

Por ahora saquemos la primera consecuencia de lo que acabamos de decir: para la filosofía del derecho es imprescindible distinguir en su objeto entre propiedades esenciales y no esenciales del mismo. Si se determinó como objeto de la Filosofía del Derecho el concepto racional del derecho y su efectuación hasta la idea del mismo, entonces esa distinción debe ser hecha en cada uno de los momentos de desenvolvimiento del concepto de derecho, es decir, en su forma original (Eidos), en su efectuación (Wirklichkeit) y en su forma final acabadamente articulada (Idea). Al mencionar que hay diferentes momentos esenciales de la idea, implícitamente se hace necesario captar las determinaciones esenciales del derecho y distinguirlas de las que no lo son, no sólo a nivel de la forma del concepto, sino también a nivel de la realización o autoefectuación del mismo y a nivel de la forma final articulada de la idea o de su realidad acabada. 
Al comienzo de la Observación al § 1 de Principios de Filosofia del Derecho, Hegel establece lo que compete como tarea a la filosofia: ocuparse de ideas y no de lo que se suele llamar "meros conceptos". 6

Quiere decir, entonces, que para que la filosofia pueda llegar a captar el concepto en su efectuación acabada no puede limitarse a intelegir sólo las propiedades esenciales de la forma conceptual, sino que es necesario que llegue a comprender las propiedades esenciales de la forma de efectuación del concepto (Verwirklichung) y las de su forma final articulada o Idea.

Como la Filosofía del Derecho tiene por objeto el concepto de derecho y su realización, debe tomar en consideración las tres perspectivas de enfoque de su objeto. Las tres, en su conjunto, constituyen el programa de exposición de la Filosofia del Derecho: mostrar el camino que recorre el concepto de derecho hasta su realización en la idea.

Pero cómo se capta las notas esenciales del concepto de derecho en el segundo momento, es decir, en el momento de su efectuación? En este punto debe echarse mano de una fina pero fundamental distinción del lenguaje hegeliano: la diferencia entre realidad (Realität) y realidad-efectiva (Wirklichkeit). ${ }^{7}$ Según esta distinción lo real no es lo mismo que real-efectivo. Lo que en un objeto es realefectivo está determinado por sus propiedades esenciales y éstas son las que constituyen, a su vez su definición conceptual. ${ }^{8}$ En Principios de Filosofía del Derecho, es decir, en la edición de 1821, § 1, Obs. Hegel nos advierte sin titubeos acerca del peligro que implica no hacer esta distinción: si se tiene a las propiedades externas, meramente reales, por reales-efectivas (wirklich) surge, en lugar de la verdad, "falsedad y engaño". Consecuentemente, afirmar que sólo el concepto es realefectivo (wirklich), significa, por lo tanto, también lo siguiente: en el mundo real (realen Welt) es real-efectivo (wirklich) sólo aquello que ha recibido la impronta del concepto, es decir, lo que está determinado por el concepto; todo lo demás es real (real) pero no real-efectivo (wirklich).

Reiteramos entonces lo que Hegel afirma en la Observación al § 1 de la obra que analizamos: todo lo que no es realidad puesta por el concepto mismo "es existencia pasajera, contingencia exterior, opinión, apariencia inesencial, falsedad, engaño etc". Desde esta perspectiva se puede afirmar consecuentemente, en cuanto al derecho, que derecho real-efectivo (wirkliches Recht) es sólo aquello que es acorde con las notas esenciales del concepto racional de derecho; y que todo

6 "Die Philosophie hat es mit Ideen, und darum nicht mit dem, was man bloße Begriffe zu heißen pflegt, zu tun, sie zeigt vielmehr deren Einseitigkeit und Unwahrheit auf, so wie daß der Begriff,.... allein es ist, was Wirklichkeit hat und zwar so, daß er sich diese selbst giebt".

7 Cfr. Philosophie des Rechts, Mitschrf. v. P. Wann., § 1, Anm., "An das vernünttige Recht schließt sich aber von selbst eine positive Sphäre an, insofern es geltendes Recht wird und äußerliche Wirklichkeit erhält”.

Glockner, Hegel-Lexikon: "Die Idee gibt an, wie die Realität durch den Begriff bestimmt ist. Alles Wirkliche ist eine Idee" (aus der Logik)... "Die Wirklichkeit ist nichst Unvernünftiges', (Nota 7, o Religiuon), "Das Zeitliche, Vergängliche existiert wohl, ist aber keine wahre Wirklichkeit, wie auch nicht die Partikularität des Subjekts, seine Wünsche, Neigungen" (Nota 18, o Plato).

8 Cfr. infra. Al respecto Principios de Filosofia del Derecho (\$ 1, Obs.) y de Philosophie des Rechts, Die Mitschrf. v. P. Wann., (§ 1, y Anm.). 
otro derecho meramente "real" (reales Recht) es sólo apariencia inesencial y, por lo tanto no es algo necesario sino contingente.

Sabemos pues que para Hegel el concepto - en este caso el del derecho tiene realidad efectiva recién cuando se puede considerar al derecho en la figura de su forma estructural final o acabada; es decir, cuando se halla totalmente desenvuelto o desarrollado; recién ahí vemos qué es lo verdaderamente real-efectivo (wirklich) del derecho y la Filosofia del Derecho debe exponer esto y, por lo tanto, debe conducir a reconocerlo. Por eso Hegel sostiene que la ciencia filosófica del derecho tiene por objeto la idea del derecho, es decir, el concepto del derecho y su realización; o sea, tiene por objeto exponer no sólo el desenvolvimiento del concepto, sino también poner de manifiesto que en ella éste se halla desarrollado o desenvuelto.

La distinción entre wirkliches Recht y reales Recht debe ser hecha en cada estadio por lo tanto también en el momento del desenvolvimiento ya logrado del concepto del derecho. Por eso agrega inmediatamente que "la configuración que se da el concepto en su realización es, para el conocimiento del concepto mismo, el otro momento esencial de la idea, que difiere de su forma de ser sólo como concepto". ${ }^{9}$

Hegel ofrece más de una justificación de esta distinción, tanto en la Filosofia del Derecho de 1821 como en las diferentes Lecciones: las razones se imponen desde el sistema mismo: el programa de la Filosofía del Derecho toma como objetivo superar la diferencia entre lo 'substancial-subjetivo' del concepto de derecho o forma conceptual (es decir, lo substancial en la subjetividad - en tanto autoconciencia de la libertad) y lo subtancial en la forma de la objetividad - en tanto realidad objetivada (Wirklichkeit) de la libertad.

Podemos suponer que la intención de Hegel es, desde la perspectiva de la idea del derecho, es decir, desde la perspectiva de la forma racional final articulada del derecho, diferenciar estadios en la realización del mismo acorde con la concepción del derecho natural racional moderno y del concepto de espiritu en tanto racional, libre y espontáneo. Y la efectuación de la idea del derecho sólo puede realizarse de manera gradual, de lo contrario se desconocería el carácter histórico de las condiciones bajo las cuales ha de desenvolverse esa idea. Dicho de otro modo: lo que Hegel trata de hacer en la Filosofia del Derecho es compatibilizar la idea de libertad del derecho racional moderno con la perspectiva histórica de las relaciones de existencia del derecho; y elige la forma de exposición que le parece más adecuada para ello, una forma que confirma, a su entender en gran medida, su concepción filosófica del tema.

La Filosofia del Derecho de Hegel adquiere así un significado muy especial. Su exposición no es aquí la tematización de un sistema de normas ius-naturales y/o su desarrollo histórico. Hegel no pretende aquí hacer comprensible el transcurrir concreto del desarrollo histónico, empírico, del derecho institucionalizado; sino que el objetivo de su exposición es más bien mostrar cómo se realiza el concepto ra-

9 Cfr. Rechtsphilosophie, Ed. Iting, Vol. II, p. 80: "Die Gestaltung, welche sich der Begriff in seiner Verwirklichung giebt, ist, zur Erkenntniß des Begriffes selbst, das andere, von der Form, nur als Begriff zu seyn, unterschiedene wesentliche Moment der Idee". 
cional del derecho, que al comienzo no estaba desenvuelto, en una forma articulada de un sistema junídico desarrollado y cómo se ubica asi en el mundo histórico.

Ahora bien, como en su obra Hegel distingue estadios de la efectuación de la libertad, puede atribuir a estos estadios, a su vez, respectivos estadios de realización del derecho; por eso Hegel sostiene: "Cada estadio del desarrollo de la idea de libertad tiene su propio derecho"; de tal manera que la Moralidad aparece como algo que tiene que ser considerado como un determinado estadio más elevado que el primero (Derecho Abstracto) del desarrollo de la idea del derecho, y el estadio que sigue, la Eticidad, como uno más elevado aún. Cada estadio del desarrollo del derecho objetivo corresponde a un estadio de derecho conceptual subjetivo y viceversa.

Podemos afirmar entonces que Hegel intenta, mediante su exposición, mostrar el autodesenvolvimiento del concepto de derecho, objeto de exposición de la Filosofia del Derecho, hacia su realidad-efectiva. En este sentido, "que el concepto tiene realidad" significa lo siguiente: recién cuando consideramos al derecho en la figura de su forma totalmente desenvuelta o desarrollada, reconocemos lo que en el derecho vigente es real-efectivo. Y Hegel pretende que la exposición de la Filosofia del Derecho conduzca hacia ese estadio de comprensión. Y en la forma que adopta para esta exposición, reiteramos una vez más, se encuentran todos los presupuestos antes mencionados: el modelo teleológico de Aristóteles: así como una semilla es comienzo y fin de un proceso de desarrollo que llega a su madurez, analógicamente, en la exposición sistemática de un concepto, el resultado debe ser, al mismo tiempo, el origen de un estadio de desenvolvimiento posterior. Esto es lo que Hegel denomina metafóricamente "estadio" (Stufe), pues cuando el desarrollo de un estadio previo a otro ha culminado, están dadas las condiciones para que comience el próximo estadio. Todo esto explica, de alguna manera, las expresiones de la Filosofía del Derecho referentes a que al final del proceso de desenvolvimiento del concepto de derecho en el Estado, la idea del derecho se halla realizada de manera efectiva y a que la Filosofia del Derecho debe exponer el autodesenvolvimiento del concepto del derecho hasta llegar a Idea.

En la forma de esta exposición sigue el siguiente esquema: comienza exponiendo la estructura interna de un concepto de derecho y la lleva hasta su extremo contrario para mostrar su contradicción, la cual es superada en una estructura más elevada. El trascurso del pensar termina cada vez en un estadio de oposición radical. Se piensa una determinacián en sí misma y por sí misma hasta el extremo de que resalte su unilateralidad y ello nos fuerza a pensar su opuesto. Lo que hace Hegel es, a partir del carácter especulativo de la reflexión transformar lo que está en contradicción en momentos del proceso de desenvolvimiento, y destacar que la unidad de los mismos es la verdad. El autodesenvolvimiento del concepto implica que cuando hemos reconocido que la cosa de la cual se trata es considerada por otros de manera diferente esto se debe a diferentes puntos de vista y perspectivas de los diferentes observadores. Entonces a uno le tiene que quedar claro que las diferentes concepciones de la cosa tienen su fundamento en la diferencia de perspectiva y de modos de consideración. Ambas cosas deben someterse a un examen crítico si es que la diferencia debe ser superada (§ 21). El proceso es similar 
a la forma de exposición de la Fenomenología, cuyo objeto es el cambio de una posición unilateral a otra y la superación de las deficiencias de ambas en una instancia superior, que ya no es unilateral..El cambio del punto de vista es el verdadero objeto de estudio.

La Filosofia del Derecho expone cómo el derecho se haya realizado, primeramente, sólo en tanto derecho abstracto y cómo, poco a poco, este derecho abandona su unilateralidad y se muestra, finalmente, en su realidad efectiva acabada. Se trata de una exposición sistemática del desarrollo de la cosa misma, el concepto de derecho, hasta su configuración acabada. Hegel lo expone así en las partes correpondientes de la Encilopedia y de la Filosofia del Derecho. Así pues, la aparente oposición entre derecho, como legalidad, y moralidad es superada por la fuerza del movimiento del concepto, el cual estatuye las relaciones de derecho. Por eso el individuo va logrando su derecho y, por lo tanto, también su acogida en el ámbito de la comunidad. El resultado de dicho movimiento es una voluntad racional, universal, la voluntad de reconocimiento, cuya realidad efectiva inmediata es la persona con capacidad juridica.

Es el concepto quien en su movimiento va salvando el hiato individualidaduniversalidad; porque en él el sujeto, en tanto tal, comprende la verdad de sí mismo, su infinitud: él es sólo real en tanto movimiento que se estatuye a sí mismo, en el movimiento mediador de devenir siempre, infinitamente, otro que sí, cabe sí mismo. El movimiento del concepto, movimiento infinito de la voluntad de reconocimiento, racional, ergo, universal, es ahora, el horizonte dentro del cual han de pensarse las relaciones de juridicidad, las relaciones éticas: la libertad de la voluntad (racional, por lo tanto, universal y sapiente) es elevada al status de principio de todas las relaciones éticas y de derecho. Ella es el resultado y, al mismo tiempo, el sujeto de dicho movimiento; la continua autodeterminación de la persona, absolutamente libre de toda determinación natural.

En el período de madurez de su pensamiento Hegel rechaza sin titubeos la idea de que la ley de la naturaleza pueda servir de modelo para el hombre a fin de normar las relaciones de convivencia. La única ley cuya impronta lleva el juego de las voluntades humanas, libres, la existencia histórica del hombre, es la ley de la libertad, de la pura unidad del yo; ley que otorga a sí mismo, no la naturaleza, sino el concepto en el infinito movimiento que va constituyendo la historia humana propiamente dicha, el tiempo de la conciencia.

En cuanto a si y cómo el concepto de derecho llega a realidad efectiva, cómo se realiza la transición de la voluntad libre a la Idea del derecho, es una cuestión abierta a la discusión aún hoy. 